\title{
Marital status and cardiovascular disease risk
}

The association between marital status and outcomes in patients with cardiovascular disease (CVD) has been controversial. In order to address this issue, Wong and colleagues ${ }^{1}$ performed a meta-analysis that included 34 studies with more than two million participants. Being unmarried, including those who never married, were divorced or widowed, was associated not only with a higher odds of CVD (OR $1.42 ; 95 \%$ CI 1.00 to 2.01 ) but also with an increased risk of coronary heart disease death (OR 1.43,95\% CI 1.28 to 1.60 ) and stroke death (OR 1.55,95\% 1.16 to 2.08 ) compared to those who were married. (figure 1) The causal mechanism for this association is unknown, although many theories abound.

In an editorial, Raparelli, Proietti, and Basili $^{2}$ put this data in the context that "While differences in health status and outcomes have been traditionally attributed to biological sex, it is now increasingly recognised that gender influences our risk of developing diseases.' (figure 2) They conclude: "It is time to apply a gender-oriented research to step forward our understanding of outcomes beyond biological factors. Gender-related factors are the missing pieces of the puzzle that should be included in the future trial design to ensure a high-quality science. A higher proportion of women must be enrolled to draw final conclusions through a well-powered sex stratify analysis.'

The proliferation of consumer smart devices capable of early detection of atrial fibrillation (AF) holds great promise for increasing the number of patients detected and treated before the occurrence of adverse events, such as stroke. Yet, many have questioned the accuracy of these new approaches for AF diagnosis. In this issue of Heart, Poh and colleagues ${ }^{3}$ evaluated the diagnostic performance of a deep learning system using photoplethysmographic (PPG) pulse waveforms for detection of AF. (figure 3) A set of over 150 thousand PPG waveforms was used to train the deep convolutional neural network (DCNN). Then this approach was tested on a data set of over 3000 smartphone-acquired PPG waveforms

Correspondence to Prof. Catherine M Otto, Division of Cardiology, University of Washington, Seattle, WA 98195, USA; cmotto@uw.edu

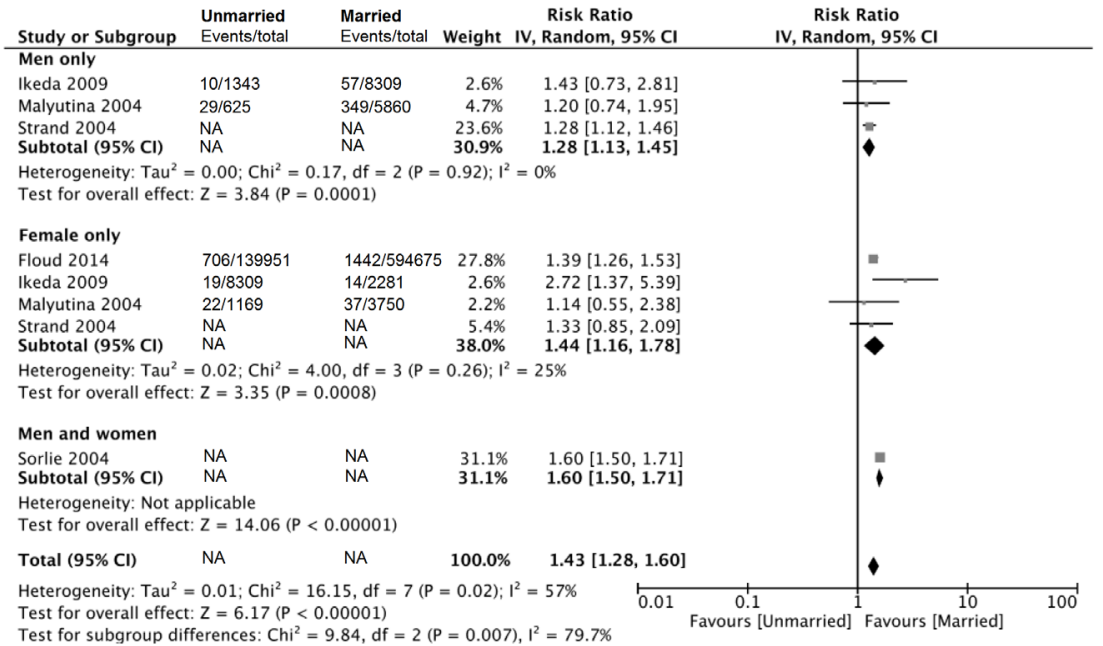

Figure 1 Forest plots of coronary heart disease (CHD) deaths in unmarried vs married in the general population.

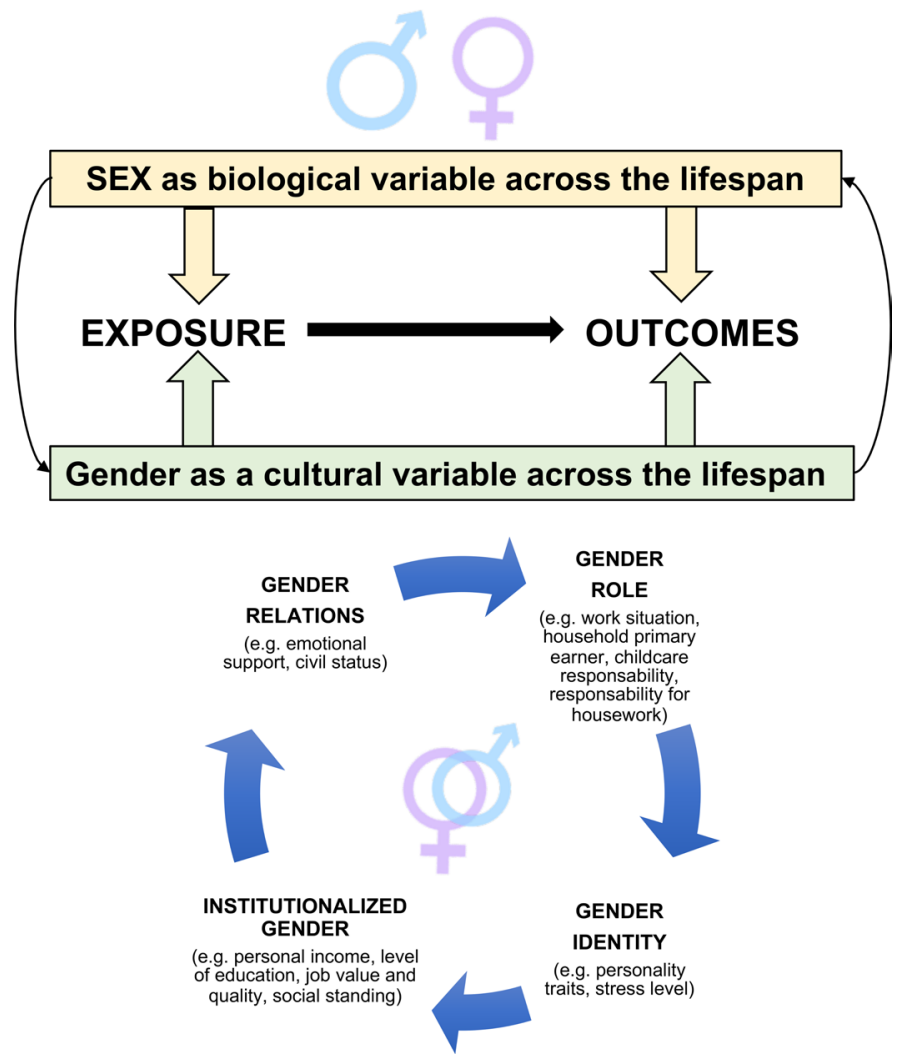

Figure 2 Sex and gender as determinants of health and disease across the lifespan.

from adults at high risk of AF (overall AF prevalence $2.8 \%$ ) compared with ECG tracings reviewed by two cardiologists. For a single 17 second PPG, the sensitivity of the DCNN was $95.2 \%$ (95\% CI $88.3 \%$ to $98.7 \%)$, specificity was $99.0 \%(95 \%$ CI $98.6 \%$ to $99.3 \%$ ), positive predictive value (PPV) was 72.7\% (95\% CI 65.1\% 
A

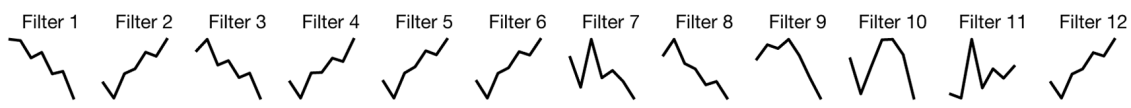

B

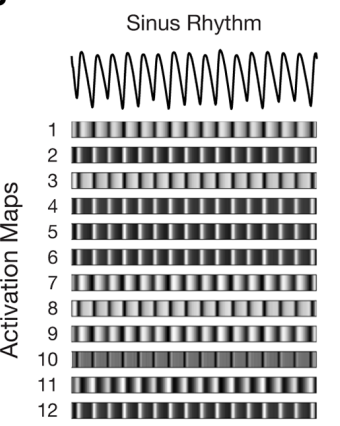

Ectopic Rhythm

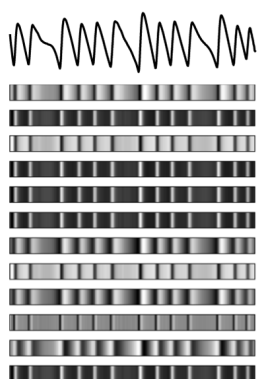

Atrial Fibrillation

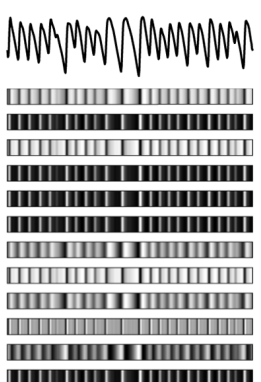

Noise

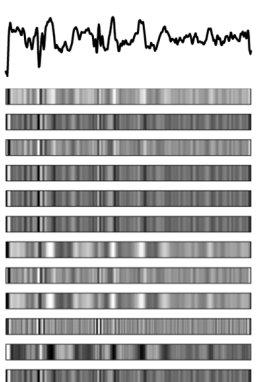

\section{C}

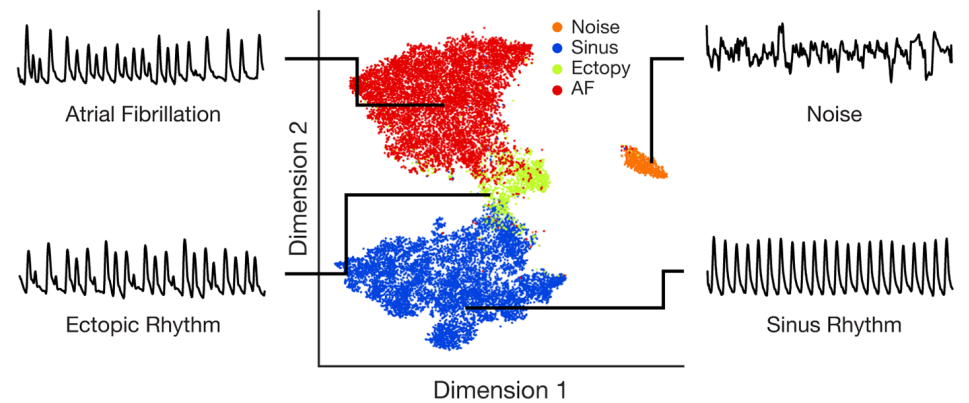

Figure 3 Visualising what the DCNN learns. (A) Learnt filters (first-layer weights) of the DCNN. (B) Layer activations. Examples of how pulse waveforms from the four different rhythm classes activate the neurons of the first convolutional layer of the DCNN. The activation maps represent the result of applying the learnt filters to the input pulse waveform. The position of a pixel in the activation map corresponds to the same position in the corresponding pulse waveform. White pixels represent strong positive activations, while black pixels represent strong negative activations at that position. (C) t-SNE visualisation. Each point in the t-SNE map represents an individual pulse waveform projected from the output of the DCNN's last hidden layer into two dimensions (of arbitrary units). The coloured clusters represent the different rhythm classes: sinus rhythm (blue), ectopy (green), noise (orange) and AF (red). Insets show examples of pulse waveforms from the different rhythm classes. AF, atrial fibrillation; DCNN, deep convolutional neural network; t-SNE, t-distributed stochastic neighbour embedding.

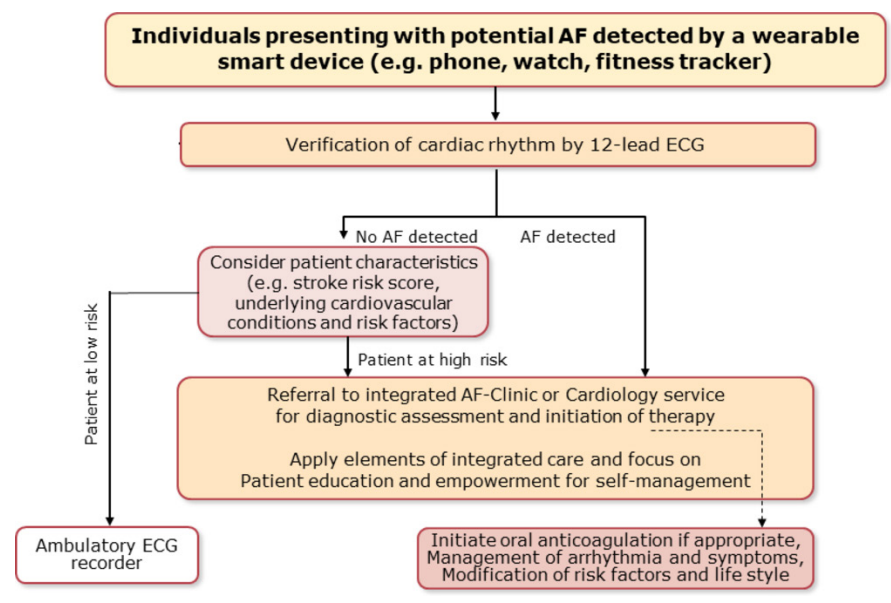

Figure 4 Potential management algorithm of atrial fibrillation (AF) detected by a wearable smart device.

to $79.3 \%)$ and negative predictive value (NPV) was 99.9\% (95\% CI 99.7\% to $100 \%)$ with even higher accuracy for longer recording periods. This DCNN approach was more accurate than six other established AF detectors.
Hendriks and colleagues ${ }^{4}$ point out in their editorial that "Smartphones and watches are easily accessible and a feature of modern society, used in all age categories and across the population spectrum. 'However, long-term application and safety of clinical decision making based on detection of AF through wearable devices is not available yet and, as the authors state, further studies would be required to both validate this approach and determine its suitability for clinical decision making with an appropriate treatment pathway in ambulatory settings.' (figure 4)

Other articles in this issue include a study of different defibrillation strategies in survivors after out-of-hospital cardiac arrest ${ }^{5}$ which emphasises the recent trend of improved survival associated with the increased use of both onsite and first responder automated external defibrillators (AEDs). The Education in Heart article provides an update on the use of triple antithrombotic therapy after acute coronary syndromes or percutaneous coronary intervention in patients on chronic oral anticoagulation. ${ }^{6}$ The Image Challenge asks you to make the diagnosis of an interesting pericardial mass based on multi-modality imaging. ${ }^{7}$

Competing interests None declared.

Patient consent Obtained.

Provenance and peer review Commissioned: internally peer reviewed.

(C) Author(s) (or their employer(s)) 2018. No commercial re-use. See rights and permissions. Published by BMJ.

Check for updates

To cite Otto CM. Heart 2018;104:1893-1894.

Heart 2018;104:1893-1894

doi:10.1136/heartjnl-2018-314336

\section{REFERENCES}

1 Wong CW, Kwok CS, Narain A, et al. Marital status and risk of cardiovascular diseases: a systematic review and meta-analysis. Heart 2018;104:1937-48.

2 Raparelli V, Proietti M, Basili S. Explanatory power of gender relations in cardiovascular outcomes: the missing piece of the puzzle. Heart 2018;104:1900-1.

3 Poh MZ, Poh YC, Chan PH, et al. Diagnostic assessment of a deep learning system for detecting atrial fibrillation in pulse waveforms. Heart 2018;104:1921-8.

4 Hendriks JM, Gallagher C, Middeldorp ME, et al. New approaches to detection of atrial fibrillation. Heart 2018;104:1898-9.

5 Zijlstra JA, Koster RW, Blom MT, et al. COSTA study group. Different defibrillation strategies in survivors after out-of-hospital cardiac arrest. Heart 2018;104:1929-36.

6 Capodanno D. Triple antithrombotic therapy after ACS and $\mathrm{PCl}$ in patients on chronic oral anticoagulation: update. Heart 2018;104:1976-83.

7 Grazzini G, Calistri L, Nardi C. Pericardial mass in a 71-year-old man. Heart 2018;104:1920. 